\title{
AJUSTE DE UNA PRUEBA DE TUBO DE COMBUSTION USANDO UN MODELO SIMPLIFICADO DE REACCIONES
}

William Navarro G. ${ }^{1 *}$, Samuel F. Muñoz N. ${ }^{2}$, Aníbal Ordoñez R. ${ }^{3}$, Hernando Bottia R. ${ }^{4}$

*A quien debe dirigirse la correspondencia

\begin{abstract}
RESUMEN
La implementación de un proceso de inyección de aire en un yacimiento de crudo pesado se fundamenta en un buen diseño experimental. Una de las principales pruebas de este diseño es la prueba de tubo de combustión, de la cual se obtienen las bases de diseño para implementar el proceso a escala piloto. Sin embargo, es de gran importancia realizar un ajuste numérico de la prueba de tubo de combustión para crear un modelo numérico a escala piloto. Usando el modelo anterior se puede evaluar el desempeño del proceso antes de su implementación directa a un piloto de campo.

Teniendo en cuenta lo anterior, en el presente estudio se construyó un modelo numérico en 1D mediante el ajuste de una prueba de tubo de combustión realizada a un crudo colombiano. Para realizar este ajuste se usaron dos reacciones con el objetivo de simular los efectos térmicos del proceso. La primera de estas reacciones tiene como único objetivo depositar el coque, el cual servirá de combustible para la segunda reacción denominada de combustión. Como resultado de la implementación del modelo anterior fue posible ajustar los picos de temperatura, el coque depositado y la velocidad del frente, variando los parámetros cinéticos de las dos reacciones.
\end{abstract}

Palabras clave: combustión in-situ, prueba tubo de combustión, modelo de simulación, velocidad frente de combustión.

\section{HISTORY MATCH OF A COMBUSTION TUBE TEST BY USING A SIMPLIFIED MODEL OF REACTIONS}

\section{ABSTRACT}

The implementation of an air injection process in a reservoir of heavy crude oil is based on a good experimental design. One of the main tests of this design is the combustion test tube, from which the design bases are obtained so that the pilot scale process can be implemented. However, it is of utmost importance to make a numerical history matching of the combustion tube test in order to create a numerical model at pilot scale. By using this model we can assess the process performance before applying it directly to a field pilot.

Considering the information above, this study is about a 1D numerical model that was built by matching a combustion tube test of Colombian crude. To do so, two reactions were used with the aim of simulating the thermal effects of the process. The first reaction's only objective is to deposit the coke, which will serve as fuel for the second reaction which is named combustion reaction. The peak temperatures, the deposited coke, and the front velocity were matched by varying the kinetic parameters of the two reactions.

Keywords: in-situ combustion, combustion tube test, simulation model, combustion front velocity.

1. Corporación Natfrac, Piedecuesta, Colombia. Email: William.navarro@natfrac.com

2. Universidad Industrial de Santander, Bucaramanga, Colombia. Email: samuel@is.edu.co

3. Instituto Colombiano del Petróleo, ECOPETROL-ICP, Piedecuesta, Colombia. Email: anibal.rodoñez@, ecopetrol.com.co

4. Instituto Colombiano del Petróleo, ECOPETROL-ICP, Piedecuesta, Colombia. Email: Hernando.bottia@ ecopetrol.com.co 


\section{INTRODUCCIÓN}

El factor de recobro promedio para los yacimientos de petróleo en el mundo es aproximadamente $35 \%$. Es decir, solo un tercio de las reservas descubiertas son producidas. En el caso colombiano, la mayoría de los yacimientos alcanzan un factor de recobro del $18 \%$. Por lo tanto, tratar de aumentar la cantidad de reservas recuperables mediante técnicas de recobro mejorado es una alternativa para suplir las necesidades energéticas del País.

Los métodos de Recobro Mejorado o Enhanced Oil Recovery (EOR), son procesos desarrollados para modificar y controlar las interacciones físicas y quimicas presentes en los yacimientos hacia condiciones más favorables para aumentar su recuperación. Para el caso de los crudos pesados, las tecnologías más favorables son los métodos térmicos, como la inyección de vapor, de agua caliente, el drenaje por gravedad asistido por vapor (SAGD), la inyección de aire (combustión In-Situ, HPAI, CAPRI), entre otras. La selección de uno de estos métodos depende de las características del yacimiento como la profundidad, la litología, la heterogeneidad de las formaciones o la geología del sistema, y por las propiedades de los fluidos presentes, particularmente la composición y la viscosidad del crudo.

En Colombia el $38 \%$ de las reservas provienen de crudos pesados y se encuentran localizadas en el Magdalena Medio y en los Llanos Orientales. Las reservas en el Magdalena Medio se encuentran a profundidades entre los 3000 y 5000 pies (ft), donde la inyección de vapor es la tecnología que se ha implementado. Por otra parte, las reservas en los Llanos Orientales se encuentran a profundidades entre los 3500 y 8000 pies (ft), con crudos móviles a condiciones de yacimiento, en los cuales la inyección de vapor es desfavorable debido a las pérdidas de calor a largo de la tubería. Es por esto, que la combustión in-situ se presenta como la mejor tecnología para incrementar el factor de recobro en yacimientos profundos de crudos pesados.

La combustión in-situ es un método de recobro mejorado. En esta técnica se inyecta aire a través de un pozo inyector con el objetivo de llevar a cabo una serie de reacciones exotérmicas entre el aceite In-Situ y el oxígeno inyectado. La energía liberada en estas reacciones es usada para disminuir la viscosidad del aceite, aumentando su movilidad, y mejorando el desplazamiento y la eficiencia de barrido, lo que se traduce en un incremento de factor de recobro.
Para implementar este proceso a nivel de campo es mandatorio llevara cabo una serie de pruebas de laboratorio con las cuales se evalúa la factibilidad de implementar el proceso de inyección de aire en un yacimiento de crudo pesado (Bottia y Gadelle, 2015). A partir de los resultados de las pruebas experimentales de tubo de combustión, se determinan los parámetros de desempeño de la prueba. Estos parámetros son el requerimiento de aire y de combustible, la velocidad del frente de combustión y los picos de temperatura alcanzados durante la prueba. La mayoría de pilotos de campo han diseñado con base a estos parámetros (Sarathi, 1999).

Para escalar los resultados obtenidos de laboratorio a escala de campo se suelen usar tanto modelos analíticos como modelos numéricos de simulación. Dentro de los modelos analíticos más utilizados están los presentados por autores como Nelson y McNeil (1961), Gates y Ramey (1980) y Moore, Laureshen, Mehta y Ursenbach (1999). Estos métodos han sido usados para el diseño y desarrollo de dos de los proyectos de combustión in situ más exitosos del mundo: el del campo Suplacu de Barcau en Rumania (Panait-Patica, Serban y Ilie, 2006) y el de los campos Santhal y Balol en la India (Adabala, Ray y Gupta, 2007). Aunque los modelos analíticos han sido usados con éxito en la actualidad existen diversos esfuerzos encaminados a desarrollar una metodología que permita el escalamiento numérico de este proceso.

El uso de modelos numéricos a escala de laboratorio proporciona una herramienta para ajustar la prueba de tubo de combustión, y evaluar los parámetros de desempeño de la prueba bajo diferentes condiciones, no realizadas en el laboratorio. Uno de los parámetros de ajuste es la velocidad del frente de combustión, la cual es constante en la prueba de tubo, esto es debido a que el área de sección transversal al flujo se mantiene constante durante la prueba. Así mismo por las condiciones de la prueba el flujo va en una sola dirección desde el pozo inyector de aire al pozo productor. Lo que se aleja de la idealidad cuando se piensa en más de una dimensión; la dirección del flujo de aire y por ende la de la velocidad del frente de combustión dependerá de la heterogeneidad del medio poroso y de la existencia de canales de permeabilidad que favorecen el flujo en determinadas direcciones. El uso de modelos numéricos a escala piloto de campo permite estudiar el comportamiento del proceso frente a variaciones en los parámetros de yacimiento y parámetros operacionales, antes de su implementación directa a escala piloto. Con lo anterior se reduce el riesgo de un fracaso al estudiar las condiciones bajo las cuales el proceso será exitoso. 
Una de las limitaciones al usar los modelos numéricos a escala de campo es la imposibilidad de escalar el modelo cinético de reacciones. Sin embargo, el uso de un modelo cinético simplificado de reacciones que contemple dos reacciones; una reacción para depositar el coque y una única reacción de combustión han sido implementadas en los simuladores para reducir el problema del escalamiento de la cinética de la reacción (Dayal et al., 2012, Priestley et. al., 2013). La simplificación del número de reacciones del modelo cinético, así como la simplificación del número de componentes del modelo de fluido y el aumento del tamaño de celdas impactará de forma directa en la reducción de los tiempos de cómputo.

Teniendo en cuenta lo anterior se realizó el ajuste de una prueba de tubo combustión para un crudo pesado colombiano, usando el esquema de reacciones simplificado propuesto por Zhu (2011). Con este esquema de reacciones el modelo cinético puede ser escalado a modelos numéricos de campo sin importar el tamaño de las celdas. Lo anterior es posible debido a que el coque que se deposita, mediante la primera reacción, no depende de la temperatura del bloque de la celda. De esta forma se garantiza que el combustible siempre estará disponible para que reaccione con el oxígeno inyectado y se genere la reacción de combustión. El calor liberado de esta reacción de combustión producirá un incremento de la temperatura y una reducción de la viscosidad del crudo.

\section{METODOLOGÍA}

En el presente trabajo de investigación, se contó con los resultados experimentales de una prueba de tubo de combustión aplicada a un yacimiento de crudo pesado colombiano.

La metodología que se desarrolló fue la siguiente. Primero se analizaron los resultados experimentales de la prueba de tubo para identificar el periodo estable de propagación del frente de combustión. Para dicho periodo se estimó la velocidad del frente de combustión y la masa de coque depositada. La velocidad del frente se calculó a partir del tiempo en el cual se alcanzó una temperatura de $400{ }^{\circ} \mathrm{C}$ en los diferentes perfiles de temperatura de las termocuplas. La masa de coque depositado fue calculada con base en los volúmenes de monóxido y dióxido de carbono producidos durante el periodo estable y siguiendo la metodología propuesta por Sarathi (1999).
Posteriormente se obtuvo un modelo cinético simplificado con dos reacciones. La primera reacción se encargará de depositar el coque y la segunda reacción se encargará de consumir el coque en una única reacción de combustión. Los coeficientes estequiométricos de la primera reacción fueron estimados con base en la cantidad de coque depositada de la prueba y siguiendo la metodología propuesta por Zhu (2011). Los coeficientes estequiométricos de la reacción de combustión fueron obtenidos a partir de la metodología propuesta por Sarathi (1999).

Finalmente se incorporó el modelo cinético simplificado en un modelo numérico en $1 \mathrm{D}$ para realizar el ajuste experimental de la prueba de tubo de combustión. Se ajustaron los picos máximos de las termocuplas, la velocidad del frente y el coque depositado. Los picos máximos de temperatura durante el periodo estable fueron ajustados al promedio obtenido experimentalmente. Los picos de temperatura, así como la velocidad del frente fueron ajustados a partir de los parámetros cinéticos de las reacciones. La masa de coque depositada fue ajustada variando el coeficiente estequiométrico del coque de la reacción número uno.

\section{ANÁLISIS DE RESULTADOS}

Los resultados obtenidos del presente estudio se dividieron en tres secciones; en la primera sección se calcularon los parámetros de desempeño de la prueba experimental, en la segunda sección se creó el modelo cinético simplificado y en la tercer se realizó el ajuste numérico de la prueba experimental.

\section{PRUEBA DE TUBO DE COMBUSTIÓN}

Una prueba de tubo de combustión para un crudo pesado colombiano fue suministrada por el Laboratorio de Inyección de Aire del ICP. Las características generales de la prueba se pueden observar en la Tabla 1.

Los registros de temperatura fueron obtenidos de 15 termocuplas localizadas a lo largo del tubo y separadas $12 \mathrm{cms}$ una de otra.

El cronograma de la prueba fue el siguiente: a los 0:0 minutos se inició la inyección de Nitrógeno, el cual se extendió por un periodo de 137 minutos, posteriormente se inició la inyección de aire, la cual finalizó a los 1133 minutos, cuando el frente alcanzó la termocupla 14. 
Tabla 1. Características Generales de la prueba Laboratorio de Inyección de Aire-ICP.

\begin{tabular}{lcc}
\hline CONDICIONES DEL ENSAYO & VALOR & UNIDAD \\
\hline Presión del ensayo & 1200 & {$[\mathrm{psi}]$} \\
\hline Temperatura del ensayo & 86 & {$\left[{ }^{\circ} \mathrm{C}\right]$} \\
\hline Tasa de inyección de aire & 4 & {$[\mathrm{~L} / \mathrm{min}]$} \\
\hline Fracción molar $\mathrm{O}_{2}$ inyectado & 20.94 & {$[\%]$} \\
\hline Densidad del crudo & 1.0016 & {$\left[\mathrm{~g} / \mathrm{cm}^{3}\right]$} \\
\hline \multicolumn{1}{c}{ EMPAQUETAMIENTO } & VALOR & UNIDAD \\
\hline Longitud del tubo & 180 & {$[\mathrm{~cm}]$} \\
\hline Radio interno del tubo & 4.88 & {$[\mathrm{~cm}]$} \\
\hline Porosidad & 40.7 & {$[\%]$} \\
\hline Volumen de roca & 12568.94 & {$[\mathrm{~L}]$} \\
\hline Volumen Poroso & 5.48 & {$[\mathrm{~L}]$} \\
\hline Masa de crudo & 3768 & {$[\mathrm{~g}]$} \\
Masa de agua & 633.35 & {$[\mathrm{~g}]$} \\
Crudo & 68.67 & {$[\%]$} \\
\hline Agua & 11.55 & {$[\%]$} \\
Gas & 19.78 & {$[\%]$} \\
\hline PRODUCCIÓN DE GASES & VALOR & UNIDAD \\
CO2 Producido * & 368 & {$[\mathrm{~L}]$} \\
CO Producido * & 100 & {$[\mathrm{~L}]$} \\
\hline O2 Producido * & 120 & {$[\mathrm{~L}]$} \\
\hline Total aire inyectado & 3,046 & {$[\mathrm{~L}]$} \\
\hline Total gas producido & 3,121 & {$[\mathrm{~L}]$} \\
\hline
\end{tabular}

*Periodo Estable

Los perfiles de temperatura para las 15 termocuplas se muestran en la Figura 1, la región estable, donde el frente se propaga a una velocidad constante está comprendido entre la termocupla 3 y la termocupla 13, que corresponde a un tiempo de 762.19 minutos. El pico máximo de temperatura es de $533^{\circ} \mathrm{C}$ [termocupla 8 ] y el promedio del pico máximo de temperatura de la región estable es de $510^{\circ} \mathrm{C}$.

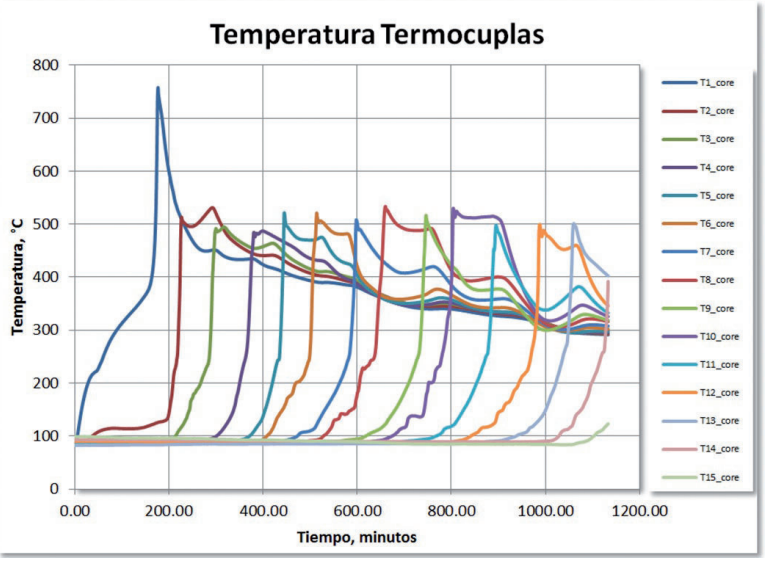

Figura 1. Perfiles de Temperatura de la Prueba
Los perfiles de los gases de combustión, así como el oxígeno producido se pueden apreciar en la Figura 2. Las altas concentraciones de $\mathrm{CO}_{2}$ [mayores al 12 \%] (Sarathi, 1999) y las bajas concentraciones de CO [menores al $4 \%$ ] (Sarathi, 1999) indican que se dieron las reacciones de oxidación a alta temperatura.

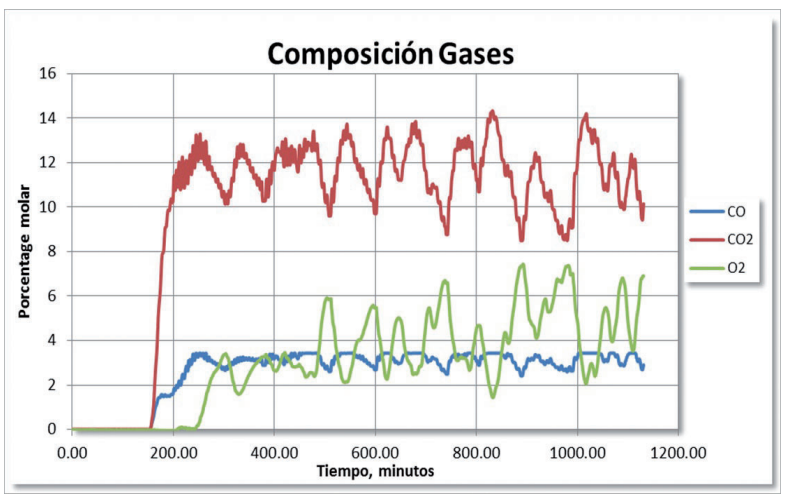

Figura 2. Perfiles de Gases de Combustión y Oxígeno

La velocidad del frente de combustión fue estimada con base en los datos de temperatura de las termocuplas No.3 a la No.13. La Figura 3 muestra la distancia y el tiempo en el cual cada termocupla alcanzó una temperatura de $400^{\circ} \mathrm{C}$, la pendiente de $0.1585 \mathrm{~cm} / \mathrm{min}[9.49 \mathrm{~cm} / \mathrm{hr}$ ] corresponde a la velocidad del frente de combustión.

Los parámetros desempeño de la prueba fueron calculados con base en la metodología definida por Sarathi (1999), en la Tabla 2 se listan los cálculos realizados.

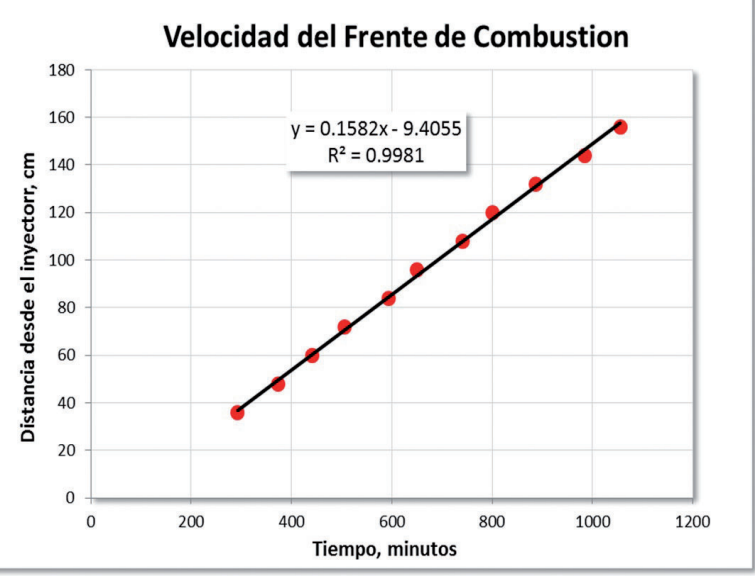

Figura 3. Velocidad del Frente de Combustión 
Tabla 2. Parámetros de desempeño de la prueba para de las Termocuplas No.3 a la No.13.

\begin{tabular}{lcc}
\multicolumn{1}{c}{ PARÁMETROS } & VALOR & UNIDAD \\
\hline Termocuplas & T3-T13 & N/A \\
\hline Volumen Roca Quemada & 8977.82 & {$[\mathrm{~cm}]$} \\
\hline $\begin{array}{l}\text { Tiempo en Quemar la Roca } \\
\text { Velocidad del Frente de }\end{array}$ & 762.19 & {$[\mathrm{~min}]$} \\
Combustión * & 9.44 & {$[\mathrm{~cm} / \mathrm{hr}]$} \\
\hline Carbono Consumido & 0.24 & {$[\mathrm{Kg}]$} \\
\hline $\begin{array}{l}\text { Oxígeno Consumido, Y } \\
\text { Hidrogeno Consumido }\end{array}$ & 81.26 & {$[\%]$} \\
\hline $\begin{array}{l}\text { Agua Formada } \\
\text { Combustible Consumido }\end{array}$ & 0.0172 & {$[\mathrm{Kg}]$} \\
\hline Relación H/C, n & 0.152 & {$[\mathrm{Kg}]$} \\
\hline Requerimiento de Combustible & 0.255 & {$[\mathrm{Kg}]$} \\
\hline Requerimiento de Aire & 0.866 & $\mathrm{NA}$ \\
\hline Relación Aire/Combustible & 28.383 & {$\left[\mathrm{Kg} / \mathrm{m}^{3}\right]$} \\
\hline $\begin{array}{l}\text { Concentración de Coque } \\
\text { depositado** }\end{array}$ & 11.955 & {$\left[\mathrm{~m}^{3}(\mathrm{~S}) / \mathrm{Kg}\right]$} \\
\hline
\end{tabular}

* Calculado a partir de la relación entre el volumen quemado, el área transversal y el tiempo en quemarlo

**Relación entre la masa de coque depositado y el volumen de roca quemada.

\section{MODELO CINÉTICO SIMPLIFICADO}

Los parámetros de los modelos cinéticos se derivan generalmente de experimentos de laboratorio como la celda de oxidación en rampa de temperatura $[R T O]$. De la prueba se obtiene un perfil de temperaturas donde es posible identificar dos regímenes de temperatura, que corresponde a dos reacciones de oxidación: a baja temperatura $[L T O]$ y a alta temperatura $[H T O]$. El coque es el principal producto de las reacciones LTO y la principal fuente de combustible para las HTO.

Existen varios modelos cinéticos que contemplan las reacciones antes mencionadas, así como las reacciones de pirolisis y craqueo térmico El modelo de Crookson (Crookston, Culham y Chen, 1979) que se compone de dos componentes de aceite [aceite pesado HO y aceite liviano $\mathrm{LO}]$ y cuatro reacciones químicas. El modelo de tres reacciones propuesto por Dechelett, Heugas, Quenault, Bothua y Christensen (2006). Representa las reacciones de pirolisis, la LTO y HTO. Dos especies distintas de combustible son definidas, los componentes Coke1 y Coke2. El modelo de Belgrave (Belgrave, Moore, Ursenbach y Bennion, 1993) que emplean una serie de reacciones que incluyen la coquización [pirolisis]. El modelo SARA (Freitag, Exelby y
Neate, 2006) que agrupan el crudo en un grupo de seudo-componentes con base a las fracciones SARA [Saturarados, Aromáticos, Resinas y Asfáltenos].

Los datos de pruebas de laboratorio [TC, RTO, ARC] proveen datos cinéticos detallados de las reacciones, pero éstos no pueden ser usados directamente en modelos numéricos de simulación (Gutierrez, Skoreyko, Moore, Mehta y Ursenbach, 2009). Esto es debido a la dependencia de la velocidad de las reacciones con la temperatura, la cual puede tener mayor representatividad en modelos de 1D, donde el tamaño de celdas puede ser del orden de los centímetros. En modelos numéricos de campo las celdas son del orden de los metros, donde las temperaturas representan el promedio de todo un bloque.

El uso de un modelo cinético que contemple dos reacciones; una reacción para depositar el coque y una única reacción de combustión han sido implementadas en modelos numéricos para reducir el problema del escalamiento de la cinética de la reacción. El modelo ha sido aplicado a un piloto del campo Balol (Dayal et al., 2012) en la India y a un sector del campo Suplacu de Barcau (Priestley et. al., 2013) en Rumania. La simplificación del esquema de reacciones a una reacción de combustión puede garantizar la generación y propagación del frente de combustión en estado estable, para ello mediante la reacción de depósito de coque se asigna un valor especifico del coque a cada una de las celdas del modelo y de esta forma habrá combustión independiente del tamaño de la celda Zhu (2011).

La reacción que se encargará de depositar el coque, consiste en un crudo original que se convertirá en un crudo más liviano $[1-X]$ y en un combustible $[Z]$ que dará inicio a la reacción de combustión. La ecuación es la siguiente:

$$
1 O I L+1 N_{2} \text { 马 }(1-X) O I L 2+1 N 2+Z C H n \ldots
$$

Al condicionar que el coque se deposite en presencia del nitrógeno se garantiza que sólo en la dirección en que se desplace el nitrógeno dentro del modelo numérico, éste se depositará. Las celdas no contactadas por el nitrógeno permanecerán con el aceite original. Esta condición es válida principalmente en modelos heterogéneos de más de dos dimensiones a escala de campo.

Para calcular el coeficiente estequiométrico del coque se parte de la masa de coque depositada en el periodo estable de la prueba de Tubo de combustión [Termocupla No.3 a la Termocupla No.13]. Se calcula el volumen de roca 
y el volumen poroso de la zona estable, para calcular la masa de crudo en dicho volumen [Eq.2]. Se estima la fracción del crudo que se convertirá en coque [Eq.3], y mediante un balance molar se obtiene el coeficiente estequiométrico para el coque [Eq.4].

$$
\begin{gathered}
W_{\text {oil }}=V_{p} . S_{\text {oil }} . \rho_{\text {oil }} \cdots \\
X=\frac{W_{\text {coque }}}{W_{\text {oil }}} \cdots \\
Z=X . \frac{M W_{\text {oil }}}{M W_{\text {coque }}} \cdots
\end{gathered}
$$

La Tabla 3 muestra los cálculos realizados para los coeficientes de la reacción que deposita el coque.

Tabla 3. Cálculos y Coeficientes Estequiométricos de la Reacción que deposita coque.

\begin{tabular}{lcl}
\multicolumn{1}{c}{ COEFICIENTE } & VALOR & UNIDAD \\
\hline Volumen de roca & 8977.82 & {$[\mathrm{cc}]$} \\
\hline Volumen poroso & 3597.41 & {$[\mathrm{cc}]$} \\
\hline Masa de Crudo & 2.47 & {$[\mathrm{Kg}]$} \\
Masa de Coque & 0.25 & {$[\mathrm{Kg}]$} \\
$\mathrm{X}$ & 0.103 & {$[$ fracción $]$} \\
$\mathrm{Z}$ & 3.786 & Adimensional \\
\hline
\end{tabular}

La reacción de combustión está representada por la reacción del coque en presencia de oxígeno para producir los gases de combustión [monóxido y dióxido de carbono] y agua. La ecuación propuesta por Benham y Poeltmann (1958) es la siguiente:

$$
a \mathrm{CH}_{n}+b \mathrm{O}_{2} \stackrel{\text { 马 }}{g}+\mathrm{cCO}_{2}+\mathrm{dCO}+e \mathrm{H}_{2} \mathrm{O} \ldots
$$

Los coeficientes de la ecuación anterior fueron obtenidos siguiendo la metodología propuesta por Sarathi (1999), ver la Tabla 4.

Tabla 4. Coeficientes Estequiométricos de la Reacción de Combustión.

\begin{tabular}{ccc} 
COEFICIENTE & VALOR & UNIDAD \\
\hline $\mathrm{a}$ & 1 & [gmol] \\
$\mathrm{b}$ & 1.109915578 & [gmol] \\
$\mathrm{c}$ & 0.786838414 & [gmol] \\
$\mathrm{d}$ & 0.21316159 & {$[\mathrm{gmol}]$} \\
$\mathrm{e}$ & 0.432992742 & {$[\mathrm{gmol}]$} \\
\hline
\end{tabular}

Una limitante que se tiene al usar estas dos reacciones es que al final del proceso no se llegará a una saturación de aceite de cero en la zona barrida. Lo anterior se debe a que no todo el petróleo original $[O I L]$ se transformara en coque, como ocurriría normalmente en un modelo cinético que contenga reacciones de pirolisis y craqueo, en donde el crudo original se transforma por completo en un combustible. Zhu (2011) recomienda ajustar las curvas de permeabilidades relativas y la tabla de viscosidad del crudo para ajustar la producción de crudo y solventar dicha limitante.

\section{AJUSTE PRUEBA DE TUBO DE COMBUSTIÓN}

Se construyó un modelo numérico en coordenadas lineales para representar el tubo de combustión con la herramienta software STARS de la compañía canadiense CMG. La longitud del tubo se dividió en 180 celdas de un (1) $\mathrm{cm}$ de espesor. A partir del área circular del tubo se obtuvo las otras dos dimensiones, asumiendo un área cuadrada. La longitud calculada para $x$ y $y$ fue de $8.646 \mathrm{~cm}$.

Para las propiedades térmicas de la roca y los fluidos fueron usadas las recomendadas por el Software. El modelo de fluidos corresponde a un crudo pesado donde sólo existe el componente crudo muerto. Las curvas de permeabilidades relativas para el sistema aguapetróleo y gas-petróleo se muestran en la Figura 4 y $\mathbf{5}$, respectivamente.

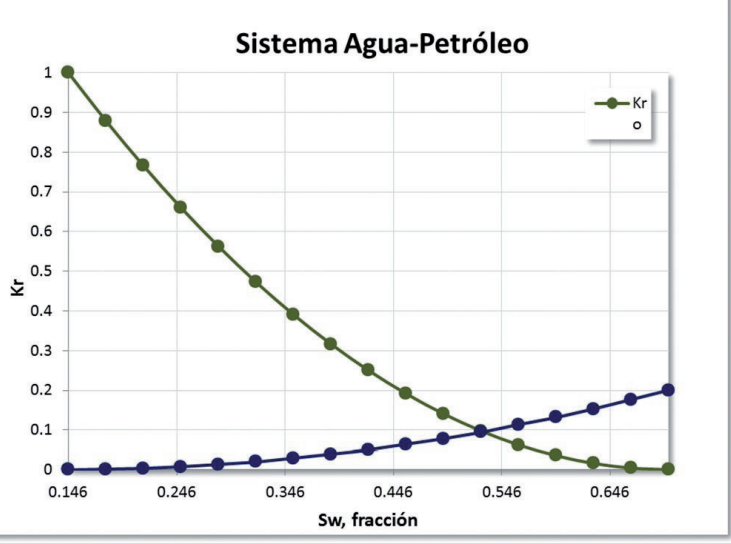

Figura 4. Curvas de permeabilidades relativas para el Sistema Agua-Petróleo. 


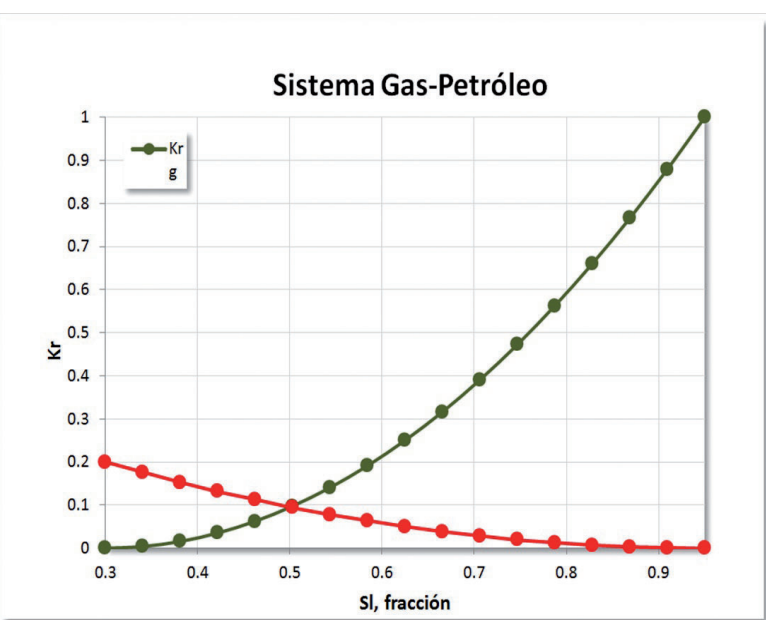

Figura 5. Curvas de permeabilidades relativas para el Sistema Gas-Petróleo.

La curva de viscosidad del crudo en función de la temperatura se obtuvo a partir de datos experimentales y mediante la ecuación de Andrade se extrapoló a otros valores de temperatura, ver Figura 6.

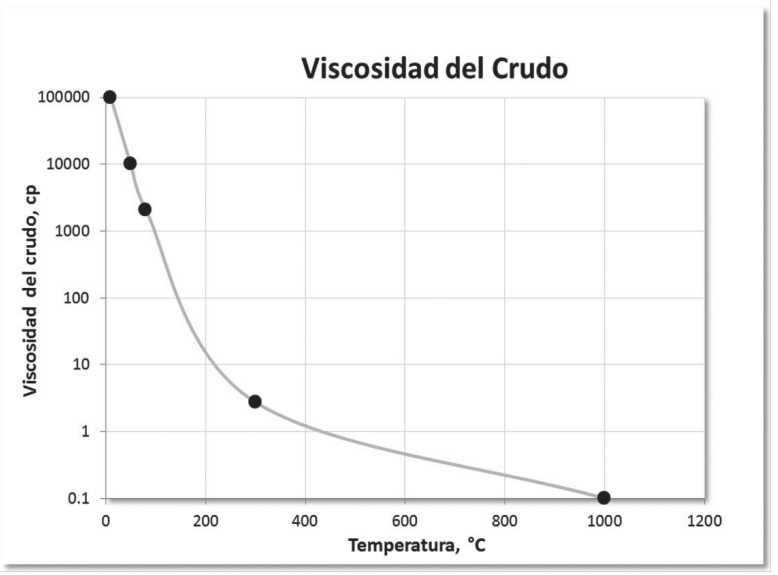

Figura 6. Viscosidad del Crudo en Función de la Temperatura.

Para lograr la ignición del crudo se definió un calentador a lo largo de la termocupla No 1. El calentador se encendió al inicio de la inyección de nitrógeno y se apagó al iniciar la inyección de aire. Este calentamiento suministró un calor de $500 \mathrm{~J} / \mathrm{min}$ por un periodo de 137 minutos hasta obtener una temperatura de $350{ }^{\circ} \mathrm{C}$ en la termocupla No.1.

\section{PARÁMETROS DE AJUSTE}

Los parámetros de ajuste para los picos de temperatura y la velocidad del frente de combustión fueron los parámetros cinéticos de las dos reacciones. Para el ajuste del coque depositado y la composición de los gases de producción se ajustó el coeficiente estequiométrico del componente coque, producido por la reacción No 1. Para el ajuste de la producción de líquidos totales se ajustó la saturación de aceite residual en el sistema gaspetróleo. Para el ajuste de la presión de inyección de aire se ajustó la permeabilidad absoluta de la roca. La Tabla 5 muestra los parámetros de ajuste.

Tabla 5. Parámetros de Ajuste del Modelo Numérico del Tubo de Combustión.

\begin{tabular}{|c|c|c|}
\hline CONDICIONES DEL ENSAYO & VALOR & UNIDAD \\
\hline Factor Pre-exponencial Reacción 1 & 100 & $1 /(\mathrm{Kpa}) / \mathrm{min}$ \\
\hline Factor Pre-exponencial Reacción 2 & 2 & $1 /(\mathrm{Kpa}) / \mathrm{min}$ \\
\hline Entalpia de la Reacción 2 & 220000 & J/gmole \\
\hline $\begin{array}{l}\text { Energía de Activación de la } \\
\text { Reacción } 2\end{array}$ & 60000 & $\mathrm{~J} /$ gmole \\
\hline $\begin{array}{l}\text { COQUE DEPOSITADO Y } \\
\text { GASES DE COMBUSTION }\end{array}$ & VALOR & UNIDAD \\
\hline $\begin{array}{l}\text { Coeficiente Estequiométrico del } \\
\text { coque de la Reacción } 1(\mathrm{Y})\end{array}$ & 3.88 & Adimensional \\
\hline LIQUIDOS TOTALES & VALOR & UNIDAD \\
\hline $\begin{array}{l}\text { Saturación de Aceite Residual en } \\
\text { Gas }\end{array}$ & 0.21 & Fracción \\
\hline $\begin{array}{c}\text { PRESION DE INYECCION DE } \\
\text { AIRE }\end{array}$ & VALOR & UNIDAD \\
\hline Permeabilidad Absoluta & 60000 & $\mathrm{mD}$ \\
\hline
\end{tabular}

\section{PICOS DE TEMPERATURA}

Los perfiles de temperatura para las termocuplas de la No.3 a la No.13 se muestran en la Figura 7. Los picos máximos de temperatura fueron ajustados al promedio obtenido experimentalmente. Las líneas discontinuas representan los datos históricos y las líneas continuas las obtenidas de la simulación. Cada par de curvas de un color representan los datos históricos y simulados de la prueba de cada termocupla, así por ejemplo las curvas de color rojo representan la termocupla No.3 y las curvas de color amarillo representan la termocupla No.13. 


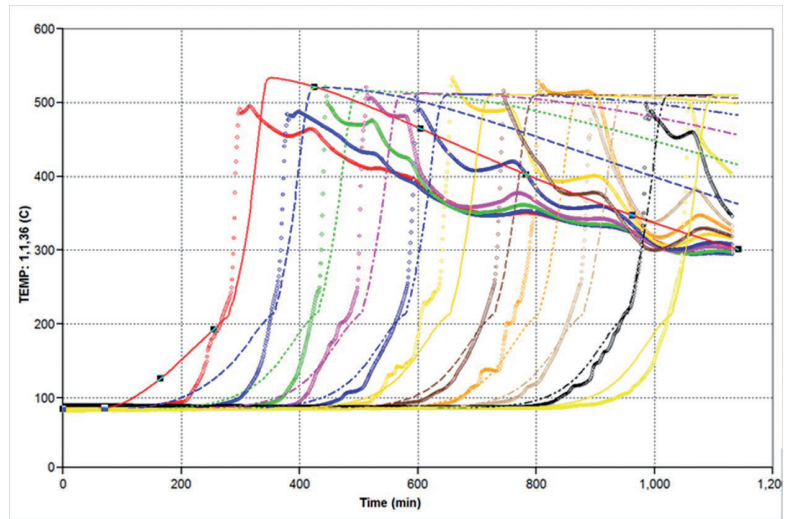

Figura 7. Perfiles de Temperatura del Modelo Numérico de la Prueba de Tubo de combustión.

\section{VELOCIDAD DEL FRENTE DE COMBUSTIÓN}

La velocidad del frente de combustión fue calculada para el periodo estable [Termocuplas No. 3 a No.13]. La Figura 8 muestra la distancia y el tiempo en el cual cada termocupla alcanzó una temperatura de $400^{\circ} \mathrm{C}$, y la pendiente de $0.1598 \mathrm{~cm} / \mathrm{min}[9.59 \mathrm{~cm} / \mathrm{hr}$ ] corresponde a la velocidad del frente de combustión. Al compararlas con los datos experimentales se obtuvo un error del $1 \%$ aproximadamente.

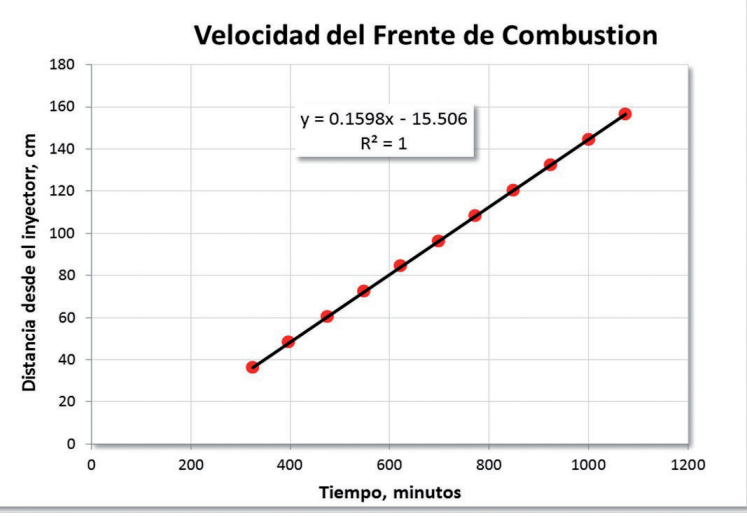

Figura 8. Velocidad del Frente de Combustión del Modelo Numérico de la Prueba de Tubo de combustión.

\section{COQUE DEPOSITADO}

La cantidad de coque depositado fue calculado con base en la masa de monóxido y dióxido de carbono producido durante el periodo estable, ver Figura 9. El coque en la termocupla No 3 se empieza a consumir a los 340 minutos y se terminó de consumir en dicha zona cuando alcanzo la termocupla No.13, es decir, a los 1104 minutos aproximadamente.

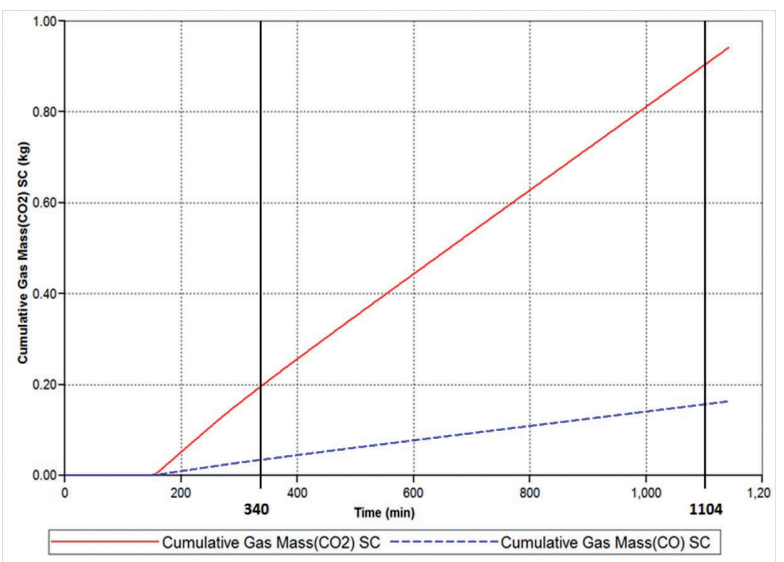

Figura 9. Masa de $\mathrm{CO} 2$ y $\mathrm{CO}$ producida durante la simulación del experimento.

La masa de $\mathrm{CO} 2$ y $\mathrm{CO}$ producido durante el periodo estable fue de $0.7069 \mathrm{Kg}$ y $0.1219 \mathrm{Kg}$, respectivamente. La masa de carbono que se deposita en forma de coque y que se consumió fue de $0.245 \mathrm{Kg}$. Al compararla con los datos experimentales se obtuvo un error del $2 \%$ aproximadamente.

\section{COMPOSICIÓN DE LOS GASES PRODUCIDOS}

Las fracciones molares de monóxido de carbono y dióxido de carbono producidas durante el periodo estable se muestran en la Figura 10.

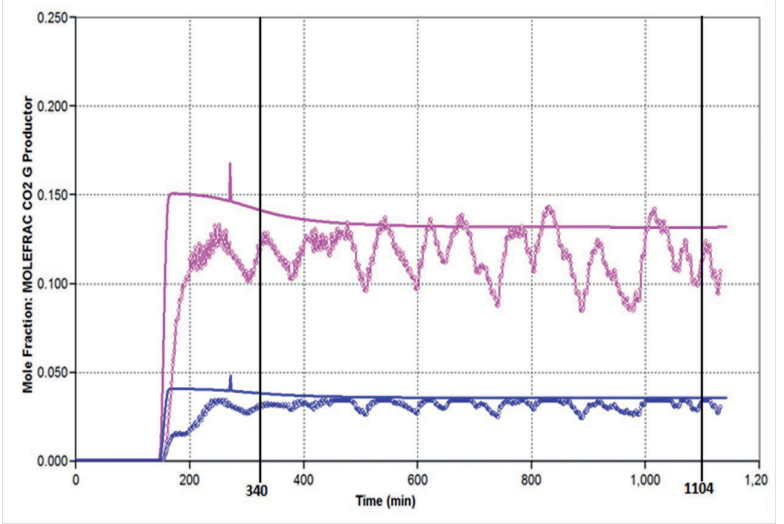

Figura 10. Ajuste de la composición de $\mathrm{CO} 2$ y $\mathrm{CO}$ de los Gases Producidos.

La línea fucsia continua es la fracción de $\mathrm{CO} 2$ producido y la línea azul continua es la fracción de CO producido. Las demás líneas representan los valores históricos. 


\section{PRODUCCIÓN DE LÍQUIDOS TOTALES}

La Figura 11 muestra el ajuste de los líquidos totales producidos. Se ajustó básicamente el inicio de la producción de fluidos y la producción acumulada de líquidos al final del periodo, ya que con un modelo simplificado de reacciones es casi imposible reproducir la tendencia histórica de la curva de producción acumulada.

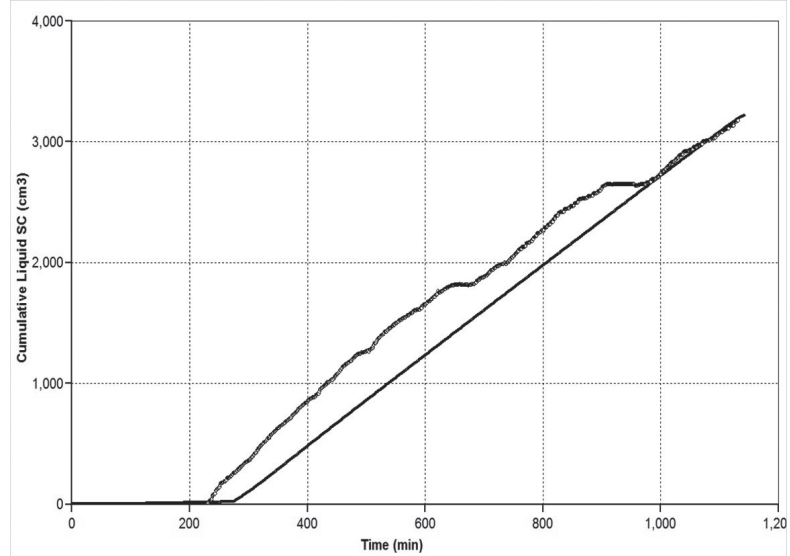

Figura 11. Ajuste de Líquidos Totales Producidos.

Debido a la imposibilidad de separar en el laboratorio el agua y el crudo producido durante la prueba, por presentarse un crudo muy emulsionado, no se pudieron ajustar los acumulados y las tasas producidas de cada fluido. Si se contara con mediciones periódicas de las tasas de agua y petróleo producidas, estas se podrían ajustar modificando las curvas de permeabilidades relativas. Dichas curvas representarían el efecto de la relación de movilidad de los fluidos tanto por cambio en la saturación como por el incremento en la temperatura.

\section{PRESIÓN DE INYECCIÓN}

La Figura 12 muestra el ajuste de la presión de inyección de aire. La línea de color negro representa los datos históricos y la línea de color gris representa los datos simulados de la prueba. El ajuste se enfocó en ajustar la máxima presión de inyección, debido a la imposibilidad de ajustar la tendencia histórica con un modelo de reacciones simplificado.

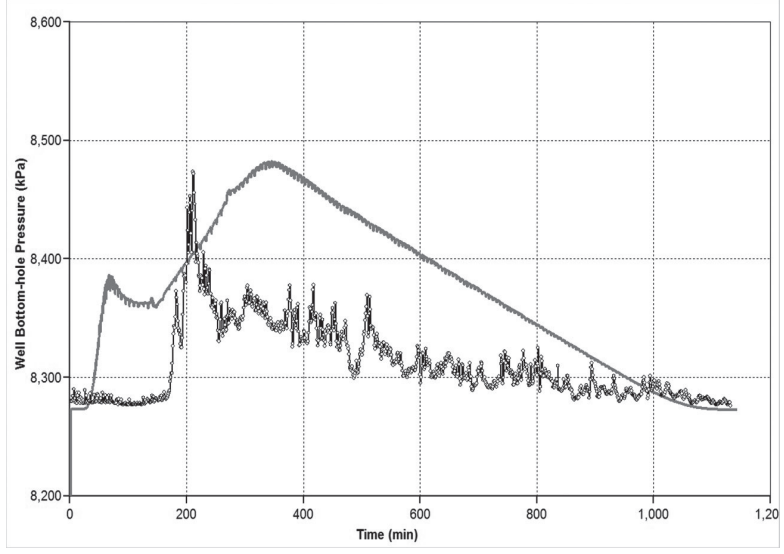

Figura 12. Ajuste de la Presión de Inyección de Aire.

\section{CONCLUSIONES}

Se logró ajustar los picos máximos de temperatura, la velocidad del frente de combustión y la masa de coque depositado de una prueba experimental de tubo de combustión, para un crudo pesado colombiano, usando un modelo cinético simplificado de reacciones. Una de estas reacciones deposita el coque y la otra reacción lo consume como combustible en una única reacción de combustión. Los picos de temperatura obtenidos en el modelo numérico corresponden al valor promedio obtenido de los datos experimentales durante el periodo estable. La velocidad del frente presentó un error del $1 \%$, al compararla con la obtenida experimentalmente. La masa de carbono depositada como coque presentó un error del $2 \%$, al compararla con los datos experimentales.

El esquema de reacciones obtenido para el modelo numérico $1 \mathrm{D}$ a escala de laboratorio puede ser usado en un modelo numérico 3D a escala piloto de campo. Donde la velocidad de la reacción que deposita el coque no estará condicionada al tamaño de las celdas del modelo. De esta forma se da solución al problema del escalamiento del modelo cinético. 


\section{REFERENCIAS}

1. Adabala, D., Ray, S. and Gupta, P. (2007). In-Situ Combustion Technique to Enhance Heavy-Oil Recovery at Mehsana, ONGC: A Success Story; SPE 105248.

2. Belgrave, J., Moore, R., Ursenbach, M., \& Bennion, D. (1993). A Comprehensive Approach to In-Situ Combustion Modeling. SPE Advanced Technology Series, pp. 98-107.

3. Benham, A., and Poettman, F. H. (1958). The Thermal Recovery Process-An Analysis of Laboratory Combustion Data. Journal of Petroleum Technology, pp. 83-85.

4. Bottia, H., \& C.Gadelle. (2015). The role of laboratory in a in situ combustion project. Features of exploration and development of unconventional hydrocarbons. Tatarstan: Editorial Ikhlas, , pp. 37-40

5. Crookston, R., Culham, W., \& Chen, W. (1979). A Numerical Simulation Model for Thermal Recovery Processes. Society of Petroleum Engineers Journal, pp. 37-58.

6. Dayal, H. S., Bhushan, B. V., Mitra, S., Pandey, V., Bhandari, A. C., \& Dwivedi, M. M. (2012). Simulation of In-Situ Combustion Process in Balol Pilot. SPE Oil and Gas India Conference and Exhibition.

7. Dechelette, B., Heugas, O., Quenault, G., Bothua, J., \& Christensen, J. (2006). Air Injection-Improved Determination of the Reaction Scheme with Ramped Temperature Experiment and Numerical Simulation. Journal of Canadian Petroleum Technology.

8. Freitag, N., Exelby, D., \& Neate, C. (2006). A SARABased Model for Simulating the Pyrolysis Reactions That Occur in High-Temperature EOR Processes. Journal of Canadian Petroleum Technology.

9. Gates, C.F. and Ramey, Jr., H.J. (1980). A Method for Engineering In-Situ Combustion Oil Recovery Projects; Journal of Petroleum Technology, Vol. 32, No. 2, pp. 285-294.
10. Gutierrez, D., Skoreyko, F., Moore, R., Mehta, S., \& Ursenbach, M. (2009). The Challenge of Predicting Field Performance of Air Injection Projects Based on Laboratory and Numerical Modelling. Journal of Canadian Petroleum Technology, pp. 23-3.

11. Moore, R.G., Laureshen, C.J., MEHTA, S.A. and URSENBACH, M.G. (1999). Observations and Design Considerations for In Situ Combustion Projects; Journal of Canadian Petroleum Technology, Special Edition, Vol. 38, No. 13, pp. 1-9.

12. Nelson, T.W. and McNeil, J.S. (1961). How to Engineer an In Situ Combustion Project; Oil \& Gas Journal, pp. 58-65.

13. Panait-Patica, A., Serban, D. and ILIE, N. (2006). Suplacu de Barcau Field: A Case History of a Successful In-Situ Combustion Exploitation; SPE 100346.

14. Priestley, A., Ruiz, J. A., Naccache, P. F., Glatz, G., \& Crecana, V. (2013). Modeling In-Situ Combustion in a Heavy Oil Field in Romania. SPE Heavy Oil Conference-Canada.

15. SARATHI, P.S. (1999). In Situ Combustion Handbook: Principles and Practices. National Petroleum Technology Office, Tulsa, Oklahoma.

16. Zhu, Z., (2011): Efficient Simulation of Thermal Enhanced Oil recovery Processes. A dissertation of Stanford University.

\section{CONVERSIÓN DE UNIDADES}

$\begin{array}{llll}1 \mathrm{~cm} & = & 0.01 \mathrm{~m} \\ 1 \mathrm{~cm}^{3} & = & 1.00 \mathrm{E}-06 \mathrm{~m}^{3} \\ 1 \mathrm{~cm} / \mathrm{hr} & = & 2.77778 \mathrm{E}-06 \mathrm{~m} / \mathrm{s} \\ 1 \mathrm{~cm} / \mathrm{min} & = & 0.000166667 \mathrm{~m} / \mathrm{s} \\ 1 \mathrm{ft} & = & 0.3048 \mathrm{~m} \\ 1 \mathrm{~g} & = & 0.001 \mathrm{Kg} \\ 1 \mathrm{~g} / \mathrm{cm}^{3} & = & 1000 \mathrm{Kg} / \mathrm{m}^{3} \\ 1 \mathrm{Kpa} & = & 1000 \mathrm{~Pa} \\ 1 \mathrm{~L} & = & 0.001 \mathrm{~m} \\ 1 \mathrm{~min} & = & 60 \mathrm{~s} \\ 1 \mathrm{Psi} & = & 6894.76 \mathrm{~Pa}\end{array}$




\section{NOMENCLATURA}

n

$\mathrm{X}$

$1-X$

Z

$W_{\text {oil }}$
Relación atómica entre $\mathrm{H}$ y $\mathrm{C}$ del coque

Fracción de aceite depositado como coque

Coeficiente estequiométrico del crudo OIL2

Coeficiente estequiométrico del coque

Masa de crudo
$V_{p} \quad$ Soil

$S_{\text {oil }} \quad$ Saturación inicial de crudo

$\rho_{\text {oil }} \quad$ Densidad del crudo

$W_{\text {coque }} \quad$ Masa de coque

$M w_{\text {oil }} \quad$ Peso molecular del crudo

$M_{\text {coque }} \quad$ Peso molecular del coque

Recepción: 22 de agosto de 2016

Aceptación: 3 de noviembre de 2016 